\title{
Could Perimenopausal Estrogen Prevent Breast Cancer? Exploring the Differential Effects of Estrogen-Only Versus Combined Hormone Replacement Therapy
}

\author{
Isaac Manyonda ${ }^{\mathrm{a}}$, Vikram Sinai Talaulikar ${ }^{\mathrm{b}, \mathrm{g}}$, Roxanna Pirhadic ${ }^{\mathrm{c}}$, John Ward ${ }^{\mathrm{d}}$, \\ Dibyesh Banerjee ${ }^{e}$ Joseph Onwude ${ }^{f}$
}

\begin{abstract}
Breast cancer is the commonest cancer among women in the western world, accounting for up to $30 \%$ of all cancers in women. There is a long-standing controversy about the potential link to hormone replacement therapy (HRT), with large observational studies suggesting that HRT increases the risk, while the Women's Health Initiative (WHI), a prospective, randomized placebo-controlled trial, has reported several times over a period of 20 years that combined (estrogen and progestogen) HRT increases the risk, while estrogen-only HRT given to women who have had a prior hysterectomy, is associated with a significantly reduced risk of developing breast cancer. Evidence from the randomized trial shows a significant reduction in both incidence of and mortality from breast cancer in women who took estrogen replacement therapy; this message needs to be presented clearly and robustly so that it can help women with decision making when considering HRT for menopause.
\end{abstract}

Keywords: Menopause; HRT; Regimens

\section{Introduction}

Traditionally, it has been thought that estrogens could play an

Manuscript submitted November 29, 2021, accepted January 17, 2022

Published online January 29, 2022

aDepartment of Obstetrics and Gynaecology, St George's University Hospitals NHS Foundation Trust/St George's, University of London, London, UK

bReproductive Medicine Unit, EGA Wing, University College London Hospital, London NW1 2BU, UK

${ }^{c}$ Faculty of Health, Education, Medicine and Social Care, Anglia Ruskin University, Chelmsford CM1 1SQ, UK

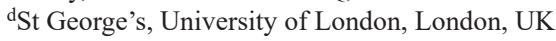

eDepartment of Surgery, St George's University Hospitals NHS Foundation Trust, London, UK

fThe Chelmsford Private Day Surgery Hospital, Fenton House, Chelmsford CM2 0PP, UK

'Corresponding Author: Vikram Sinai Talaulikar, Reproductive Medicine Unit, EGA Wing, University College London Hospital, London NW1 2BU, UK. Email: vikram.talaulikar@nhs.net

doi: https://doi.org/10.14740/jocmr4646 important role in the pathophysiology of breast cancer, firstly because the female breast contains an abundance of estrogen receptors, and secondly because of the observation that estrogens can aggravate estrogen receptor-positive breast cancer [1]. Neither are sustainable as possible explanations since firstly, breasts are only one of many organs that are suffused with estrogen receptors (others include the brain, eyes, hair, nails, the skin, fatty tissues, muscles, cartilage, bones and blood vessels), and there is no concern about estrogen-related cancer in these organs (although receptor density could explain differences). Secondly, not unexpectedly there have been women who have been diagnosed with breast cancer soon after commencing hormone replacement therapy (HRT). This of course does not prove causation, and in fact the mortality from breast cancer in these situations is actually often low [2], suggesting that the process of carcinogenesis had already started, and commencing estrogens might have actually allowed an earlier diagnosis [3]. Thus, despite being the commonest cancer in women in the developed western world, and notwithstanding the intense research activity aimed at it, the mechanism(s) by which breast cancer develops remains an enigma. There are of course a number of theories out there, including a role for endocrine disruptors [4], but these continue to be researched with no current resultant effective preventative or therapeutic interventions.

\section{The Epidemiology of Breast Cancer: Impact of HRT Versus Life-Style Factors}

In the UK, it is estimated that if 1,000 otherwise healthy women who are not on HRT and aged 50 - 60 years are followed up over 5 years, 23 of them will develop breast cancer [5]. Figure 1 illustrates that if another 1,000 such women who have an intact uterus are given combined hormone replacement therapy (cHRT), an additional four women (total 27) will develop breast cancer, while in a 1,000 who have had a hysterectomy and therefore can be given estrogen-only therapy (estrogen replacement therapy (ERT)), four fewer women (total 19) will develop the disease. Therefore, while cHRT is associated with an increased risk of breast cancer, ERT does not increase the risk, and if anything, appears to be associated with a reduced risk.

To further put the issue into perspective, the impact of life-style factors has been added (Fig. 1). Amongst smokers, 


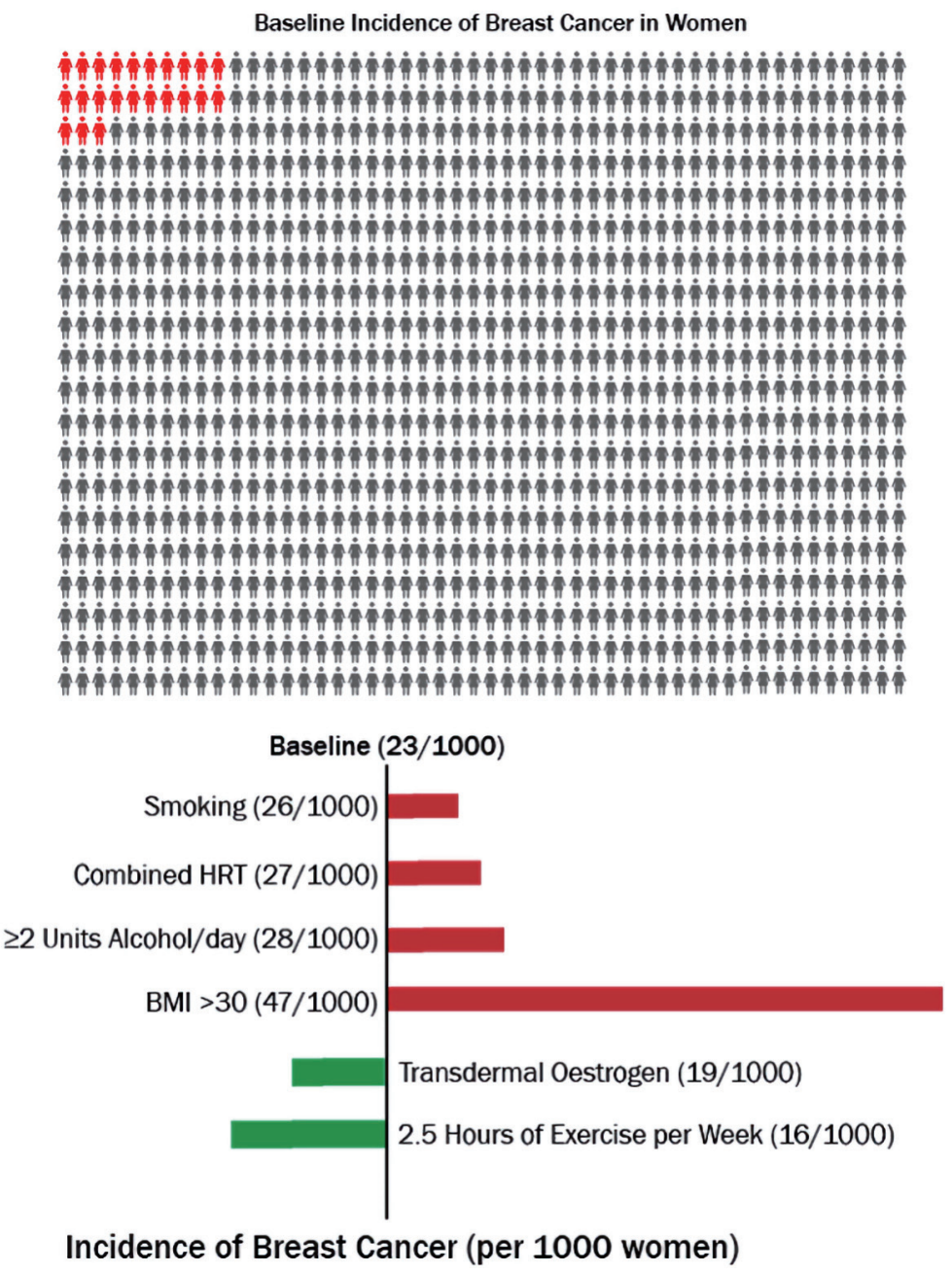

Figure 1. Risk of breast cancer associated with different modifiable risk factors over 5 years in women aged 50 - 59. HRT: hormone replacement therapy; BMl: body mass index.

there will be an additional three cases of breast cancer (total 26), while two units of alcohol per day will add five women to the afflicted (total 28), and obesity (body mass index (BMI) $>30$ ) will add a phenomenal 24 women, giving a total of 47 in 1,000. Conversely, exercise lasting $2.5 \mathrm{~h}$ per week will reduce the number by 7 (total 16). These numbers illustrate that although cHRT increases the risk of breast cancer, the increase is relatively small compared to the risk posed by other modifiable risk factors, and that the risk is amenable to even simple interventions like exercise. If phrases such as "exercise prevents breast cancer" could be used to promote women's health, could the parallel phrase "estrogen-only HRT prevents breast cancer" be acceptable? It is useful to consider what the research evidence shows.

\section{HRT and Breast Cancer: Evaluating the Evi- dence From Research}

Over the years, issues relating to HRT and breast cancer have generated huge controversy, debates and divided opinions among the healthcare professionals as well as the general public, usually resulting in women who might otherwise benefit from HRT abandoning it or being denied it by their physicians. This has often been a result of the way in which research findings have been interpreted. It is important to note that valid randomized placebo-controlled studies are traditionally regarded as the best evidence of causation between an intervention and a disease provided the comparison groups are similar at baseline. Retrospective observational studies are not useful to prove a "cause and effect relationship" as they cannot entirely eliminate bias.

\section{HRT and Breast Cancer: The Evidence From Observational Studies}

Probably the three most influential observational studies on breast cancer risk in association with HRT are the "Collaborative Re-analysis" [6] and the "Million Women" [7] studies 
Table 1. Major Observational and Interventional Studies on Risk of Breast Cancer Associated With Use of Hormone Replacement Therapy

Study and year
Breast cancer and hormone
replacement therapy: collaborative
reanalysis of data from 51
epidemiological studies of 52,705
women with breast cancer and 108,411
women without breast cancer. Beral et
al, Collaborative Group on Hormonal
Factors in Breast Cancer, 1997 [6]
Risks and benefits of estrogen plus
progestin in healthy postmenopausal
women: principal results from the
Women's Health Initiative randomized
controlled trial. Rossouw et al,
Writing Group for the Women's Health
Initiative Investigators, 2002 [9]

Breast cancer and hormonereplacement therapy in the Million Women Study. Million Women Study Collaborators, 2003 [7]

Type and timing of menopausal hormone therapy and breast cancer risk: individual participant meta-analysis of the worldwide epidemiological evidence. Collaborative Group on Hormonal Factors in Breast Cancer, 2019 [8]

Association of menopausal hormone therapy with breast cancer incidence and mortality during long-term follow-up of the Women's Health Initiative randomized clinical trials. Chlebowski et al, 2020 [10]

\section{Findings}

Among current users of HRT or those who ceased use 1 - 4 years previously, the relative risk of having breast cancer diagnosed increased by a factor of $1.023(95 \% \mathrm{CI}: 1.011-1.036 ; 2 \mathrm{P}=0.0002)$ for each year of use; the relative risk was $1.35(1.21-1.49 ; 2 \mathrm{P}=0.00001)$ for women who had used HRT for 5 years or longer (average duration of use in this group 11 years). Cancers diagnosed in women who had ever used HRT tended to be less advanced clinically than those diagnosed in never-users.

After a mean of 5.2 years of follow-up on HRT, results were: estimated HRs (nominal 95\% CIs) were as follows: heart disease, $1.29(1.02-1.63)$ with 286 cases; breast cancer, $1.26(1.00-1.59)$ with 290 cases; stroke, 1.41 (1.07 - 1.85) with 212 cases; PE, 2.13 (1.39 - 3.25) with 101 cases; colorectal cancer, $0.63(0.43$ - 0.92) with 112 cases; endometrial cancer, $0.83(0.47$ - 1.47) with 47 cases; hip fracture, $0.66(0.45-0.98)$ with 106 cases; and death due to other causes, $0.92(0.74-1.14)$ with 331 cases. Absolute excess risks per 10,000 person-years attributable to estrogen plus progestin were seven more CHD events, eight more strokes, eight more PEs, and eight more invasive breast cancers, while absolute risk reductions per 10,000 person-years were six fewer colorectal cancers and five fewer hip fractures. The absolute excess risk of events included in the global index was 19 per 10,000 person-years.

Half the women in study had used HRT; and 9,364 incident invasive breast cancers and 637 breast cancer deaths were registered after an average of 2.6 and 4.1 years of follow-up, respectively. Current users of HRT at recruitment were more likely than never users to develop breast cancer (adjusted relative risk $1.66(95 \% \mathrm{CI}: 1.58-1.75), \mathrm{P}<0.0001)$ and die from it $(1.22(1.00-1.48), \mathrm{P}=0.05)$. Incidence was significantly increased for current users of preparations containing estrogen only (1.30 (1.21 - 1.40), $\mathrm{P}<0.0001)$, estrogen-progestogen (2.00 (1.88 - 2.12), $\mathrm{P}<0.0001)$, and tibolone (1.45 (1.25 - 1.68), $\mathrm{P}<0.0001)$, but the magnitude of the associated risk was substantially greater for estrogen-progestogen than for other types of HRT $(\mathrm{P}<0.0001)$.

During prospective follow-up, 108,647 postmenopausal women developed breast cancer at mean age 65 years (SD: 7); 55,575 (51\%) had used HRT. Among women with complete information, mean hormone therapy duration was 10 years (SD: 6) in current users and 7 years (SD: 6) in past users, and mean age was 50 years (SD: 5) at menopause and 50 years (SD: 6) at starting HRT. Every HRT type, except vaginal estrogens, was associated with excess breast cancer risks, which increased steadily with duration of use and were greater for estrogen-progestogen than estrogen-only preparations. Among current users, these excess risks were definite even during years 1 - 4 (estrogen-progestogen RR: $1.60,95 \%$ CI: 1.52 - 1.69; estrogen-only RR: 1.17, $1.10-1.26$ ), and were twice as great during years 5 - 14 (estrogen-progestogen RR: 2.08, 2.02 - 2.15; estrogen-only RR: 1.33, 1.28 - 1.37).

Among 27,347 postmenopausal women who were randomized (baseline mean (SD) age, 63.4 years (7.2 years)), after more than 20 years of median cumulative follow-up, mortality information was available for more than $98 \%$. Estrogen (CEE) $0.625 \mathrm{mg}$ daily alone compared with placebo among 10,739 women with a prior hysterectomy was associated with statistically significantly lower breast cancer incidence with 238 cases (annualized rate: $0.30 \%$ ) vs. 296 cases (annualized rate: 0.37\%; HR: 0.78 ; 95\% CI: $0.65-0.93$; $\mathrm{P}=0.005)$ and was associated with statistically significantly lower breast cancer mortality with 30 deaths (annualized mortality rate: $0.031 \%$ ) vs. 46 deaths (annualized mortality rate: $0.046 \%$; HR: $0.60 ; 95 \% \mathrm{CI}: 0.37-0.97 ; \mathrm{P}=0.04)$. In contrast, CEE $0.625 \mathrm{mg}$ daily plus progestogen (MPA) $2.5 \mathrm{mg}$ daily compared with placebo among 16,608 women with a uterus was associated with statistically significantly higher breast cancer incidence with 584 cases (annualized rate: $0.45 \%$ ) vs. 447 cases (annualized rate: $0.36 \%$; HR: $1.28 ; 95 \% \mathrm{CI}: 1.13-1.45 ; \mathrm{P}<0.001$ ) and no significant difference in breast cancer mortality.

HR: hazard ratio; $\mathrm{Cl}$ : confidence interval; PE: pulmonary embolism; CHD: coronary heart disease; SD: standard deviation; CEE: conjugated equine estrogen; HR: hazard ratio; RR: risk ratio; MPA: medroxyprogesterone acetate.

and the meta-analysis by the Collaborative Group on Hormonal Factors in Breast Cancer in 2019 [8] (Table 1 [6-10]). The Collaborative Re-analysis of data from 51 epidemiological studies of 52,705 women with breast cancer and 108,411 women without breast cancer [6] concluded that the risk of having breast cancer diagnosed is increased in women using HRT and increases with increasing duration of use. This effect is reduced after cessation of use of HRT and has largely, if not 
wholly, disappeared after about 5 years. The general implication was that HRT caused breast cancer. This re-analysis of observational studies included retrospective and prospective studies. Shapiro et al (2011) [11] tested the re-analysis on the basis of standard criteria when an observational study asserts causality. They examined the following factors: time order, bias, confounding, statistical stability, strength of association, dose-duration response, internal and external consistency and biologic plausibility. They concluded that the causality link reached by the Collaborative Re-analysis was defective. This independent report means that the Collaborative Re-analysis has low scientific validity because of serious significant epidemiological faults.

The Million Women's Study [7] was a prospective cohort of UK women aged 50 to 64 years invited to undergo screening mammography at 3 -yearly intervals. Among 828,923 postmenopausal women who were current users of HRT and followed for an average of 2.6 years, the study concluded that current use of HRT was associated with increased breast cancer incidence and mortality, and the effect was substantially greater for estrogen-progestogen combinations (cHRT) than for other types of HRT. Again, the general implication was that HRT caused breast cancer. This prospective study had many methodological shortcomings and the most cogent was that it did not exclude breast cancers that appeared within 1 year, as they were most likely to have been present at baseline. Shapiro et al (2011) [12] also tested the Million Women's Study on the basis of standard criteria when an observational study asserts causality. They examined the following factors: time order, bias, confounding, statistical stability, strength of association, dose-duration response, internal and external consistency and biologic plausibility. They concluded that HRT may or may not increase the risk of breast cancer, but the Million Women's Study did not establish that it does. The causality link was unreliable because of defects in quality of design, execution, analysis and interpretation. They commented that size alone did not guarantee that the findings are reliable. This independent report means that the Million Women's Study again has little scientific validity because of serious epidemiological faults. However, the Million Women's Study differentiated that there was a lower risk of incident breast cancer between women on ERT or tibolone compared to women on cHRT [7].

The recent meta-analysis from the Collaborative Group on Hormonal Factors in Breast Cancer in 2019 [8] again reviewed the evidence from epidemiological rather than interventional studies, and results are not in keeping with the randomized Women's Health Initiative (WHI) trials which have recently reported that women receiving estrogen post hysterectomy had a lower long-term risk of breast cancer.

\section{HRT and Breast Cancer: The Evidence From Randomized Controlled Studies}

The WHI studies are without doubt the most influential of any of the prospective, placebo-controlled randomized trials of HRT and breast cancer risk. The research comprised two randomized trials that included 27,347 postmenopausal women, mean age 63.4 (standard deviation (SD) 7.2), all of whom had a negative mammogram and no prior breast cancer at baseline. Enrolment took place from 1993 to 1998, with participants being contacted for follow-up every 6 months through 2005 and annually from then on, and mortality data being gathered from follow-up and the National Death Index. In the first trial, which included 16,608 women with a uterus, 8,506 women received $0.625 \mathrm{mg}$ /day of conjugated equine estrogen (CEE) plus 2.5 $\mathrm{mg}$ /day of medroxyprogesterone acetate (MPA), while 8,102 received placebo. In the second trial, the women had had a hysterectomy and therefore did not need MPA: 10,739 women were randomized to $0.625 \mathrm{mg} /$ day CEE $(5,310$ women) while 5,429 women received placebo. The first trial ended in 2002 after a median intervention period of 5.6 years, and the second trial ended in 2004 after a period of 7.2 years.

\section{Outcomes from cHRT versus placebo}

This WHI study reported six times. The first five reports between 2002 and 2009 [9, 13-17] were based on a randomized controlled trial. The fifth report incorporated a prospective observational cohort with follow-up for a further 8 years [18].

The second report focused more on women who developed breast cancer after an average of 5.6 years in the randomized controlled trial. Shapiro et al (2011) [17] highlighted that there was a degree of contamination with 331 women in the concurrent estrogen replacement trial who still had a uterus, who were unblinded and added to the combined estrogen plus progesterone group versus placebo trial. Nevertheless, for all breast cancers, the hazard ratio (HR) was 1.24 (95\% confidence interval (CI): 1.02 - 1.50) when 8,507 women aged 50 - 79 years who received cHRT were compared to 8,102 similar women who received placebo.

Based on the WHI randomized controlled trial only and not the sixth report [18], which was a combination of randomized controlled trial and follow-on observational study, the WHI studies of cHRT versus placebo show a causal link between cHRT and incidence of breast cancer. The long-term results confirm this causality [19].

\section{Outcomes from ERT versus placebo}

This WHI study reported five times. The first report was held to be valid, because apart from similar baseline characteristics, there were similar proportions of un-blinding, similar discontinuation rates, and similar proportions of those who were prescribed HRT by their own doctors. The second report focused more on women who developed breast cancer after an average of 7.1 years in the randomized controlled trial. In the "intention to treat analysis", there was a $23 \%$ non-significant reduction in the risk of breast cancer compared to placebo (relative risk ratio (RR): $0.77 ; 95 \% \mathrm{CI}: 0.59$ - 1.01). However, in an "as treated analysis" which satisfies time order, minimizes detection bias and where confounding was unlikely, there was a $33 \%$ significant reduction in the risk of breast cancer compared to placebo (RR: $0.67 ; 95 \%$ CI: 0.47 - 0.97). These results 
persisted after 10.7 years.

Shapiro et al (2011) [17] stated that the evidence suggests that unopposed estrogen does not increase the risk of breast cancer and may even reduce it. The latter possibility, however, was initially based on statistically borderline evidence. The long-term results confirm this lack of a causal link between unopposed estrogen and incidence of breast cancer, and that unopposed estrogens do not increase the risk of breast cancer, in fact reducing it [19].

What is unique and remarkable about the WHI studies of HRT and breast cancer risk is that once again, after a median of 20.3 years of follow-up, and with mortality data now available for more than $98 \%$ participants, outcomes have been reported/updated in JAMA in July 2020 [10]. In the trial involving 16,608 women with a uterus, 8,506 were randomized to receive $0.625 \mathrm{mg}$ daily of CEE plus $2.5 \mathrm{mg}$ daily of MPA, and 8,102 with placebo. In the trial involving 10,739 women with prior hysterectomy, 5,310 were randomized to receive 0.625 mg daily of CEE alone, and 5,429 with placebo. The CEEplus-MPA trial was stopped in 2002 after 5.6 years' median intervention duration, and the CEE-only trial was stopped in 2004 after 7.2 years' median intervention duration. The key findings were as follows: 1) CEE alone (ERT) was associated with fewer cases of breast cancer ( 238 cases, annualized rate $0.30 \%$ ), compared with placebo (296 cases, annualized rate 0.37\%; HR: 0.78; 95\% CI: $0.65-0.93$; P = 0.005). Furthermore, CEE alone was also associated with lower mortality (30 deaths, annualized mortality rate $0.031 \%$ ), compared with placebo (46 deaths, annualized mortality rate $0.046 \%$; HR: 0.60 ; 95\% CI: 0.37 - 0.97; $\mathrm{P}=0.04)$; 2) In contrast, CEE plus MPA (cHRT) was linked with more cases of breast cancer (584 cases, annualized rate $0.45 \%$ ) than placebo ( 447 cases, annualized rate $0.36 \%$; HR: 1.28 ; 95\% CI: $1.13-1.45 ; \mathrm{P}<0.001)$. In regard to mortality, there was no statistically significant difference between CEE plus MPA (71 deaths, annualized mortality rate $0.045 \%$ ) and placebo (53 deaths, annualized mortality rate 0.035\%; HR: 1.35; 95\% CI: $0.94-1.95 ; \mathrm{P}=0.11$ ).

Thus, the WHI studies show that taking CEE alone for up to 7 years confers protection against breast cancer for at least 20 years, while taking the combination therapy CEE and MPA for just 5 years increases the risk, which persists for at least 20 years. Is it the combination therapy, or is it the progestogen alone, that causes the breast cancer? While the answer is not as clear cut as might be imagined, it should be remembered that estrogens increase glandular tissue, while it is the progestogens that cause mitosis of breast tissue - cancer represents uncontrolled mitosis.

\section{Implications of the New Insights on the Preven- tative Benefits of Estrogen}

The evidence now compels a paradigm shift from the traditional thinking that estrogen could cause breast cancer to a recognition that it actually prevents the disease, and that when the disease does occur (no preventative intervention achieves a $100 \%$ preventative effect), it is often picked up early and mortality is reduced by up to $44 \%$. Therefore, rather than being left to fear estrogen, the majority of perimenopausal women should be offered the hormone, on its own in those who have had a hysterectomy, and with a progestogen-releasing intrauterine device in those with an intact uterus. This cheap and safe hormone also has other preventative potential. Estrogen prevents osteoporosis [20], a condition that can lead to bone fractures with a major impact on quality of life, increased mortality and is a significant drain on NHS resources [21]. In the premenopausal phase women enjoy protection against cardiovascular disease, but soon catch up with men after the menopause: estrogen given as HRT protects against cardiovascular disease in women [22]. A gender difference in favor of women in terms of infection rates and mortality has been clearly observed in a variety of pandemic-prone viral infections including coronavirus disease 2019 (COVID-19) [23], severe acute respiratory syndrome (SARS) [24] and Middle East respiratory syndrome (MERS) [25]; and estrogen has been implicated either by boosting the immune system, both innate and adaptive, and also by direct action on cell types such as the vascular endothelium [26, 27]. Estrogen reduces the risk of colorectal cancer [28], and there is increasing evidence from in vitro, animal and human experimentation that it may protect against dementia [29]. Finally, estrogen is the most effective intervention in the treatment of menopausal symptoms [30,31], such as hot flushes and night sweats, vaginal dryness, shedding hair and dry skin, emotional lability to name but a few of the symptoms women may suffer in the menopausal transition.

Modern regulated HRT combines hormones in various doses and routes to make the preparations safe and effective. Minor side effects associated with HRT include irregular bleeding, nausea, breast tenderness, headaches, bloating and skin sensitivity for transdermal preparations. These are usually temporary and settle after 2 - 3 months. Transdermal hormone preparations minimize the risk of thrombosis that has been traditionally associated with oral hormone preparations.

At a time when there is an emphasis on prevention as a major strategic approach to improve the nation's health [32], estrogen should be recognized for its huge potential.

\section{Conclusions}

It is generally accepted that contemporary best clinical practice should be evidence-based, with the best clinical evidence coming from randomized clinical trials. The WHI study of ERT versus placebo in women with a prior hysterectomy is a most robust piece of research: prospective, randomized, placebocontrolled and with a 20-year follow-up, which now compels a direct interpretation of its finding, namely that exposure to exogenous estrogen (ERT) prevents breast cancer. This is of profound importance, not only in relation to the prevention of the most common cancer in women in the western world, but also because estrogen, whilst being cost-effective and well-tolerated also has other preventative properties against osteoporosis and cardiovascular disease, to name but two. If the medical professionals are struggling with the required paradigm shift in their attitude to ERT, then a well-designed, adequately powered, prospective randomized trial with so many spin-offs is 
eminently doable. Results could be available in less than 10 years, and if such results support those from the WHI, then there would be no further arguments or debate.

\section{Acknowledgments}

None to declare.

\section{Financial Disclosure}

No funding was received for preparation of this manuscript.

\section{Conflict of Interest}

All authors (IM, VST, RP, JW, DB, JO) report no conflict of interest.

\section{Author Contributions}

JO and IM wrote the first draft of the manuscript. JO, IM, RP, VT, DB and JW contributed to analysis of the studies and writing of the final draft of the manuscript.

\section{Data Availability}

The authors declare that data supporting the findings of this study are available within the article.

\section{References}

1. Yue W, Wang JP, Li Y, Fan P, Liu G, Zhang N, Conaway $\mathrm{M}$, et al. Effects of estrogen on breast cancer development: Role of estrogen receptor independent mechanisms. Int J Cancer. 2010;127(8):1748-1757.

2. Mikkola TS, Savolainen-Peltonen H, Tuomikoski P, Hoti F, Vattulainen P, Gissler M, Ylikorkala O. Reduced risk of breast cancer mortality in women using postmenopausal hormone therapy: a Finnish nationwide comparative study. Menopause. 2016;23(11):1199-1203.

3. Onwude JL. Response to hormone replacement therapy and the breast. BMJ. 2002;323:1381.

4. Macon MB, Fenton SE. Endocrine disruptors and the breast: early life effects and later life disease. J Mammary Gland Biol Neoplasia. 2013;18(1):43-61.

5. Women's Health Concern. Factsheet: Understanding the risks of breast cancer. A comparison of lifestyle risk factors versus Hormone Replacement Therapy (HRT) treatment. March; 2017, Buckinghamshire, UK. https:// thebms.org.uk/wp-content/uploads/2016/04/WHC-UnderstandingRisksofBreastCancer-MARCH2017.pdf.

6. Collaborative Group on Hormonal Factors in Breast Cancer. Breast cancer and hormone replacement thera- py: collaborative reanalysis of data from 51 epidemiological studies of 52,705 women with breast cancer and 108,411 women without breast cancer. Collaborative Group on Hormonal Factors in Breast Cancer. Lancet. 1997;350(9084):1047-1059.

7. Beral V, Million Women Study Collaborators. Breast cancer and hormone-replacement therapy in the Million Women Study. Lancet. 2003;362(9382):419-427.

8. Collaborative Group on Hormonal Factors in Breast Cancer. Type and timing of menopausal hormone therapy and breast cancer risk: individual participant meta-analysis of the worldwide epidemiological evidence. Lancet. 2019;394(10204):1159-1168.

9. Rossouw JE, Anderson GL, Prentice RL, LaCroix AZ, Kooperberg C, Stefanick ML, Jackson RD, et al. Risks and benefits of estrogen plus progestin in healthy postmenopausal women: principal results From the Women's Health Initiative randomized controlled trial. JAMA. 2002;288(3):321-333.

10. Chlebowski RT, Anderson GL, Aragaki AK, Manson JE, Stefanick ML, Pan K, Barrington W, et al. Association of menopausal hormone therapy with breast cancer incidence and mortality during long-term follow-up of the women's health initiative randomized clinical trials. JAMA. 2020;324(4):369-380.

11. Shapiro S, Farmer RD, Seaman H, Stevenson JC, Mueck AO. Does hormone replacement therapy cause breast cancer? An application of causal principles to three studies: Part 1. The Collaborative Reanalysis. J Fam Plann Reprod Health Care. 2011;37(2):103-109.

12. Shapiro S, Farmer RD, Stevenson JC, Burger HG, Mueck AO. Does hormone replacement therapy cause breast cancer? An application of causal principles to three studies. Part 4: the Million Women Study. J Fam Plann Reprod Health Care. 2012;38(2):102-109.

13. Anderson GL, Chlebowski RT, Rossouw JE, Rodabough RJ, McTiernan A, Margolis KL, Aggerwal A, et al. Prior hormone therapy and breast cancer risk in the Women's Health Initiative randomized trial of estrogen plus progestin. Maturitas. 2006;55(2):103-115.

14. Heiss G, Wallace R, Anderson GL, Aragaki A, Beresford SA, Brzyski R, Chlebowski RT, et al. Health risks and benefits 3 years after stopping randomized treatment with estrogen and progestin. JAMA. 2008;299(9):1036-1045.

15. Prentice RL, Chlebowski RT, Stefanick ML, Manson JE, Pettinger M, Hendrix SL, Hubbell FA, et al. Estrogen plus progestin therapy and breast cancer in recently postmenopausal women. Am J Epidemiol. 2008;167(10):12071216.

16. Chlebowski RT, Kuller LH, Prentice RL, Stefanick ML, Manson JE, Gass M, Aragaki AK, et al. Breast cancer after use of estrogen plus progestin in postmenopausal women. N Engl J Med. 2009;360(6):573-587.

17. Shapiro S, Farmer RD, Mueck AO, Seaman H, Stevenson JC. Does hormone replacement therapy cause breast cancer? An application of causal principles to three studies: part 2. The Women's Health Initiative: estrogen plus progestogen. J Fam Plann Reprod Health Care. 2011;37(3):165-172. 
18. Shapiro S, Farmer RD, Mueck AO, Seaman H, Stevenson JC. Does hormone replacement therapy cause breast cancer? An application of causal principles to three studies: part 3. The Women's Health Initiative: unopposed estrogen. J Fam Plann Reprod Health Care. 2011;37(4):225230.

19. Chlebowski RT, Garnet L, Anderson GL, Aragaki AK, et al. Long-term influence of estrogen plus progestin and estrogen alone use on breast cancer incidence: The women's health initiative randomized trials. San Antonio Breast Cancer Symposium. December 13, 2019.

20. Gambacciani M, Levancini M. Hormone replacement therapy and the prevention of postmenopausal osteoporosis. Prz Menopauzalny. 2014;13(4):213-220.

21. Leal J, Gray AM, Prieto-Alhambra D, Arden NK, Cooper C, Javaid MK, Judge A, et al. Impact of hip fracture on hospital care costs: a population-based study. Osteoporos Int. 2016;27(2):549-558.

22. Lobo RA, Pickar JH, Stevenson JC, Mack WJ, Hodis HN. Back to the future: Hormone replacement therapy as part of a prevention strategy for women at the onset of menopause. Atherosclerosis. 2016;254:282-290.

23. Jin JM, Bai P, He W, Wu F, Liu XF, Han DM, Liu S, et al. Gender differences in patients with COVID-19: focus on severity and mortality. Front Public Health. 2020;8:152.

24. La Vignera S, Cannarella R, Condorelli RA, Torre F, Aversa A, Calogero AE. Sex-specific SARS-CoV-2 mortality: among hormone-modulated ACE2 expression, risk of venous thromboembolism and hypovitaminosis D. Int J Mol Sci. 2020;21(8):2948.
25. Hui DS, Azhar EI, Kim YJ, Memish ZA, Oh MD, Zumla A. Middle East respiratory syndrome coronavirus: risk factors and determinants of primary, household, and nosocomial transmission. Lancet Infect Dis. 2018;18(8):e217e227.

26. Manyonda IT, Pereira RS, Makinde V, Brincat M, Varma RT. Effect of 17 beta-oestradiol on lymphocyte subpopulations, delayed cutaneous hypersensitivity responses and mixed lymphocyte reactions in post-menopausal women. Maturitas. 1992;14(3):201-210.

27. Pirhadi R, Sinai Talaulikar V, Onwude J, Manyonda I. Could Estrogen Protect Women From COVID-19? J Clin Med Res. 2020;12(10):634-639.

28. Johnson JR, Lacey JV, Jr., Lazovich D, Geller MA, Schairer C, Schatzkin A, Flood A. Menopausal hormone therapy and risk of colorectal cancer. Cancer Epidemiol Biomarkers Prev. 2009;18(1):196-203.

29. Simpkins JW, Perez E, Wang X, Yang S, Wen Y, Singh M. The potential for estrogens in preventing Alzheimer's disease and vascular dementia. Ther Adv Neurol Disord. 2009;2(1):31-49.

30. Maclennan AH, Broadbent JL, Lester S, Moore V. Oral oestrogen and combined oestrogen/progestogen therapy versus placebo for hot flushes. Cochrane Database Syst Rev. 2004;4:CD002978.

31. Fait T. Menopause hormone therapy: latest developments and clinical practice. Drugs Context. 2019;8:212551.

32. Public Health England. PHE Strategy 2020-25. Protectine and improving the nation's health. September 2019. London, UK. 